\title{
A NOVEL AND INNOVATIVE METHOD FOR DESIGNING OF RF MEMS DEVICES
}

\author{
Deepak Kumar Sinha ${ }^{1}$, Sandhya Save ${ }^{2}$ \\ ${ }^{1}$ M.E, EXTC (Pursuing), Electronics \& Telecommunication Department, Thakur College of Engineering \& \\ Technology, Maharashtra, India \\ ${ }^{2}$ Head of Department, Electronics Engineering Department, Thakur College of Engineering \& Technology, \\ Maharashtra, India
}

\begin{abstract}
The design complexity of the RF MEMS devices is increasing with fast rate, which require more accurate designing and simulation techniques. With upcoming technologies accurate simulation capability is a necessity for designing smaller chips. When devices are designed at nano scale, it presents a number of unique challenges. As the scale of the individual device decreases and the complexity of the physical structure increases, the nature of the device characteristics depart from those obtained from many of the classically held modeling concepts. Furthermore, the difficulty encountered in performing measurements on these devices means we have to put more emphasis on the results obtained from theoretical characteristics. Modeling also allows new device structures to be rigorously investigated prior to fabrication. This paper reports a novel and innovative method of design and simulation of MEMS based inductor by the method of co-simulation.
\end{abstract}

Keywords: Micro Electro Mechanical System, Radio Frequency, Co-Simulation, COMSOL Multiphysics, SOLIDWORKS, Computer Aided Design, Integrated Circuit

\section{INTRODUCTION}

The word Micro Electromechanical System came into existence when the integration of electronic and mechanical components was possible on the same silicon chip. MEMS technology is the most important and challenging technology of the 21 st Century and it can revolutionize both industrial and consumer products by combining silicon based microelectronics with micromachining technology. The major application areas of this technology are RF circuits, chemical sensors, biosensors, aerospace, consumer electronics, etc. MEMS-based devices can range in size from a few micrometers to millimeters. The MEMS industry is can work as the driving force for next generation systems and subsystems.

As the consumer applications have been realized by using microwave technology due to the decrease of power consumption and high power handling capacity, the production has increased a lot. It has motivated the boost in processing techniques for MEMS systems over the years, and we are increasingly leaning towards their applications in System on chip (SoC), optical systems and many more.

\subsection{RF-MEMS}

RF MEMS is one of the most important areas of MEMS technology which is growing at the fastest rate with commercial applications. In the field of communication engineering, RF MEMS can be specifically designed for mobile phones and other wireless communication devices such as radar and antennas.
The MEMS technology can enhance the performance and reliability of such devices while reducing their size simultaneously.

The important elements designed by using MEMS technology which are used for tuning are inductors, capacitors, filters, resonators and switches etc.

At microwave frequencies, micro machined lumped components can replace distributed components with flexibility in integration as well as improvement in bandwidth.

\subsection{Introduction to RF MEMS Inductor}

The performance of various RF circuits mainly depends on the spiral inductor used in it. When it comes to design of various radio frequencies (RF) matchingnet works, load circuits of voltage controlled oscillators, filters, mixers etc., the inductor is a basic component and plays important role in overall performance[1].

The quality factor is an important parameter that decides the behavior of high performance RF circuit. With the advancement of silicon processing technologies, huge improvements have been made to the size and speed of the transistor. Similarly RF circuits operating at higher frequencies will also benefit from small-size, high Q-factor inductors [7]. 
For example, the LC tank, in which the quality factor Q of thespiral inductor determines the bandwidth and the resonance impedance of the LCtank.

Another example is the band pass filter (BPF) built with inductors andcapacitors, in which the quality factor of the spiral inductor determines theinsertion loss. In low noise amplifiers (LNA), figure of merit (FoM) decides the overall performance and it depends on the quality factor of the spiral.In voltage controlled oscillators (VCO), high-Qspiral inductors reduce both DC power consumption and phase noise [2].

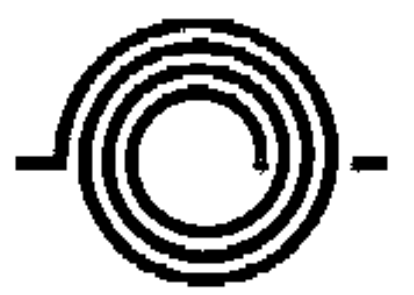

(a)

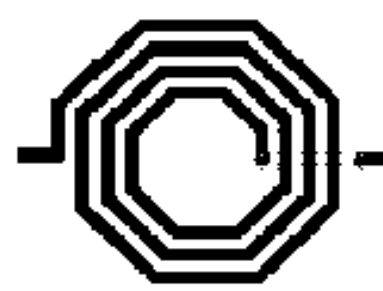

(c)

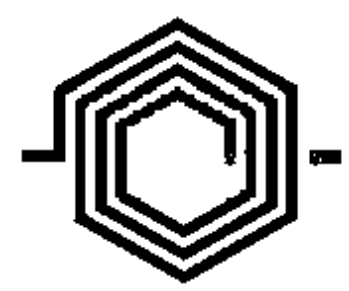

(b)

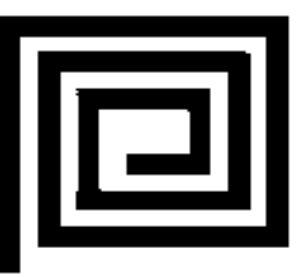

(d)
Fig 1.1 (a) Circular Spiral (b) Hexagonal Spiral (c) Octagonal Spiral (d) Rectangular Spiral MEMS Inductors

\section{REQUIREMENT OF INNOVATIVE METHOD}

\subsection{Requirement of RF MEMS Device Simulation}

Device simulation is a cost-effective method of determining whether a new technology is worth developing. Once that decision has been made, simulation can substitute for many costly matrix experiments that are normally required to optimize a new process and device structure.

This is especially important in MEMS technologies because of the statistical and complex nature of device design. Function must be guaranteed for the large number of devices in modern electronic systems. It is impossible to produce enough experimental hardware to test even the most critical combinations of parameters and structures. Large numbers of devices and significant variations in structure from device to device make a statistical design imperative.

Another advantage that simulation offers is certainty of the structure and physical parameters of the device; i.e., device design information can be derived before a new fabrication process has been stabilized and the device can be optimized early in the product development cycle.
A final advantage is that the internal operation of any device can be easily examined through multidimensional simulation experimental techniques which can do this only approximately and for very few parameters. Modeling devices is always challenging because the scaling down of dimension and the nonlinear nature of device physics requires simulations which are computationally expensive.

\subsection{Limitation of Simulation Software}

We use different software for design and simulation of MEMS devices. When we want to design a MEMS device model in 3D, we have to adopt two approaches. Either we have to use complex Python based complex programs or we have to use the designing tool available in that simulation software itself.

When it comes to python based programming, it requires very good knowledge of programming techniques. It may be very time-consuming process to write a program for complex designs. When it comes to use the designing tools present in the simulation software, we can design 2D and 3D models both.

But designing of complex structures in $3 \mathrm{D}$ is not always possible. Sometimes we have to use the technique of rotation of the $2 \mathrm{D}$ layout designed on a work plane to get the $3 \mathrm{D}$ model. But for complex structured devices, it is not possible to generate such 2D layouts and hence it creates a limitation of the simulation software in designing the complex 3D models.

\subsection{Requirement of Co-Simulation}

The different subsystems forming a coupled problem are modeled and simulated by using Co-simulation in a distributed manner. Hence, while modeling is being done on the subsystem levels we do not carefor the coupled problem at that time. Furthermore, by running the subsystem, the coupled simulation is carried out in a black-box manner. During the simulation the subsystems will exchange data.

In simple words, related to the work carried out here, it can be defined as the "integration of best features of designing software and best features of simulating software at a time." As the device size decreases, various undesired effects are prevailing, which can be reduced if better and accurate design method is available. Hence, it is required to have an advanced designing tool which can be used at the simulating software platform as well.

By using the method of co-Simulation, we can design and simulate any 3D MEMS devices easily and efficiently. 


\section{PROPOSED METHOD FOR DESIGNING}

Designing the MEMS Structure in 3D using SOLIDWORKS Software

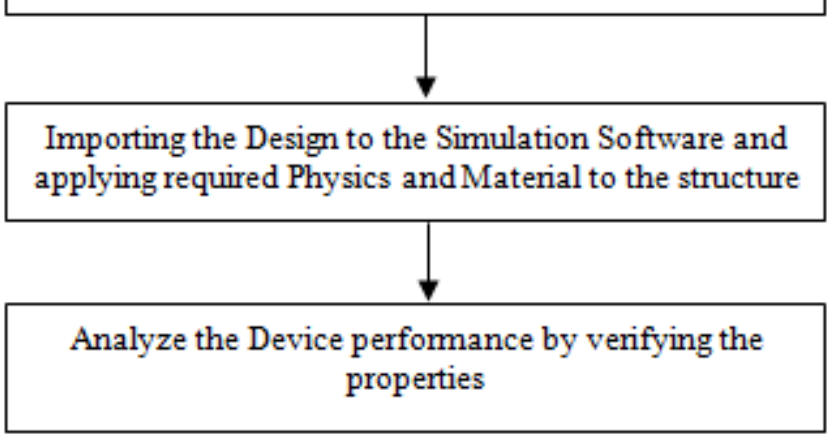

Fig 3.1 Methodology of Proposed project

\section{IMPLEMENTATION DETAILS}

\subsection{Design of MEMS based Rectangular Spiral}

\section{Inductor using SOLIDWORKS Software}

Step 1:-Select the plane of work and draw the appropriate layout of the structure by using sketch button as shown under.

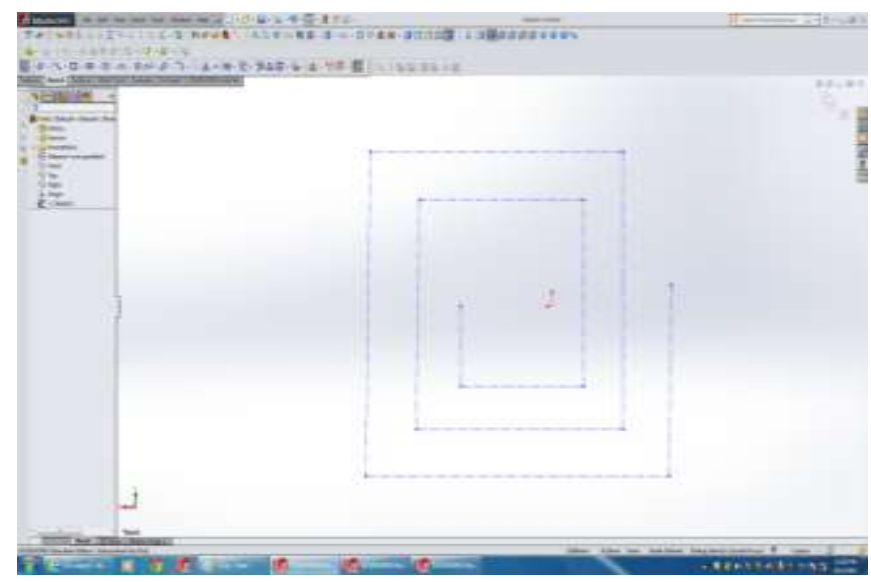

Fig 4.1 Sketching of the plane

Step 2:-Specify the dimensions and trim the entity.

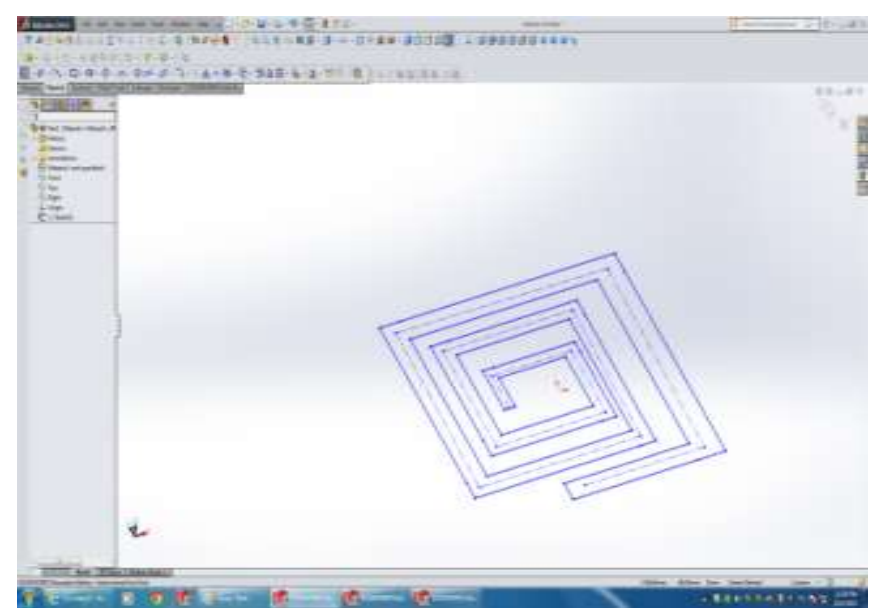

Fig 4.2Trimming the structure
Step 3:-Then extrude the structure to give it the desired width of desired dimensions.

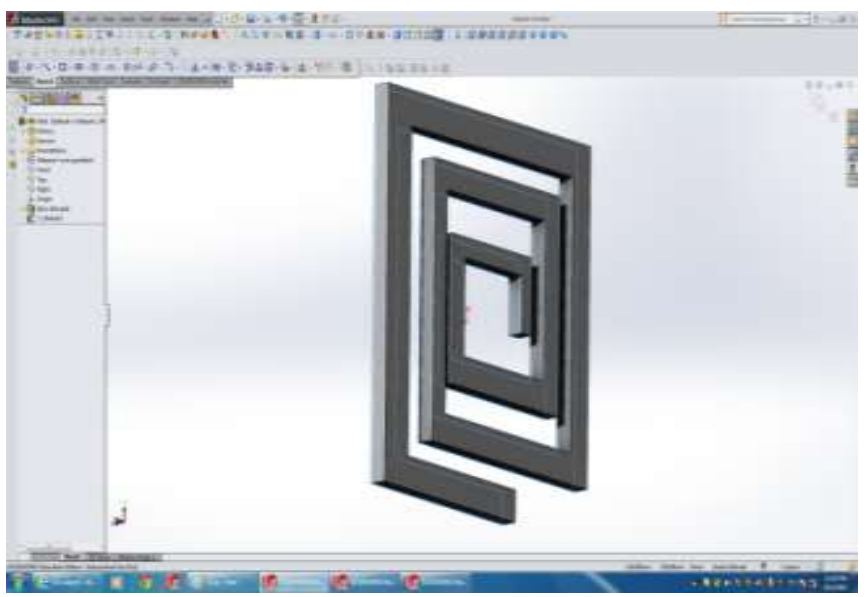

Fig 4.3Extruding of the layer

Step 4:-Sketch and extrude again to take one additional edge required for the structure as shown in the figure below.

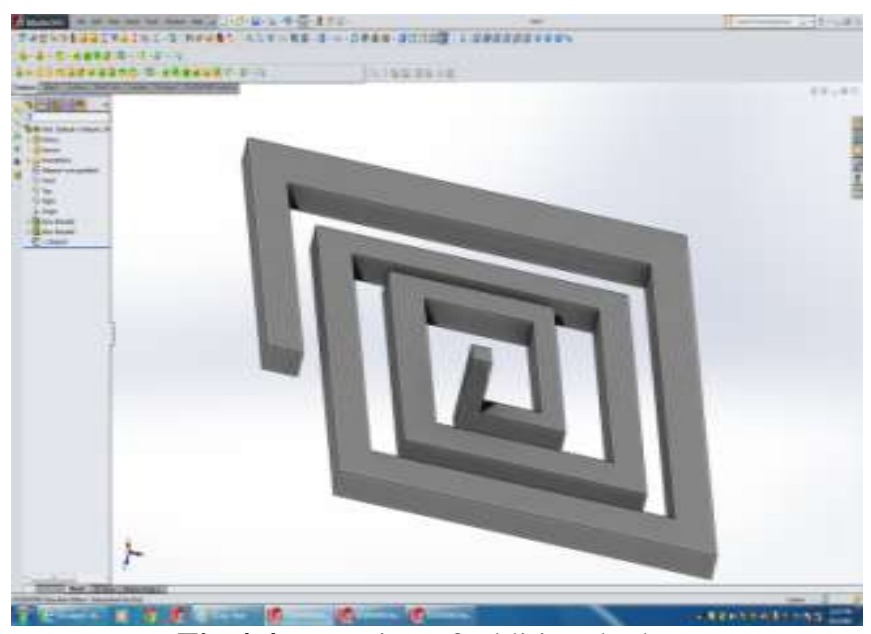

Fig 4.4Formation of additional edge

Step 5:-Repeat step4 to get the final structure as shown under.

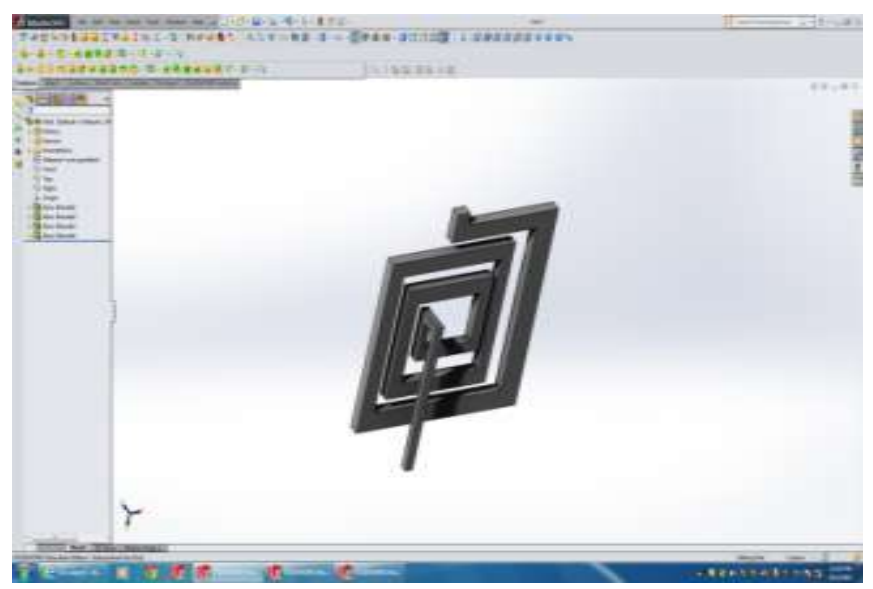

Fig 4.5Final structure of the MEMS Inductor

Step 6:-Save the design with the extension mane as .igs or .iges. 


\subsection{Co-Simulation with COMSOL Multiphysics}

Step1:-Open COMSOL Multiphysics, select 3D model and then specify the physics as semiconductor (Semi) and then choose the study as Stationary. Then import the IGS/IGES file saved in SolidWorks. Then click on Build all button.

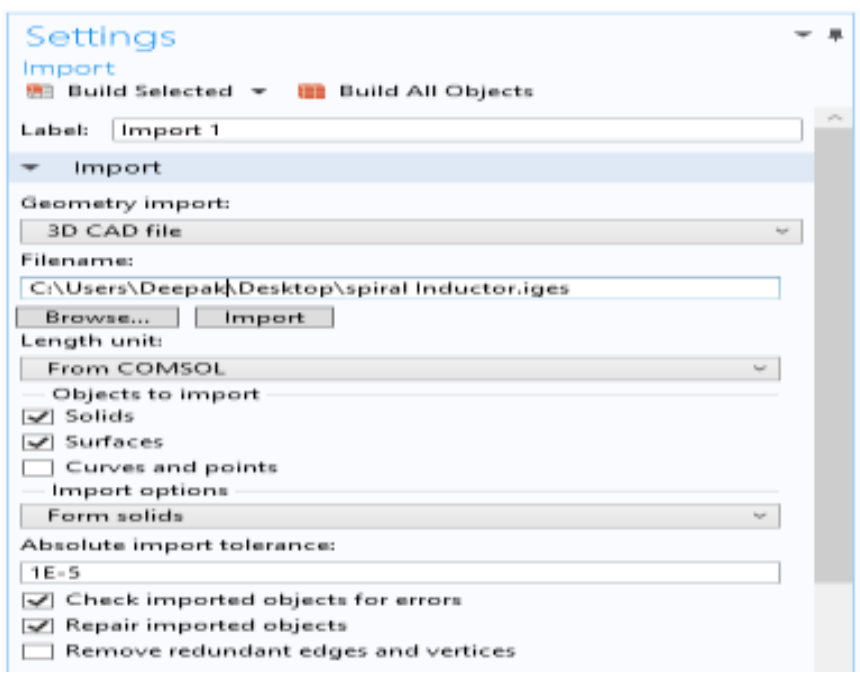

Fig 4.6 Importing the IGS File to COMSOL

Step2:-After Clicking on Build all we get the structure in the graphics window as shown in the figure below.

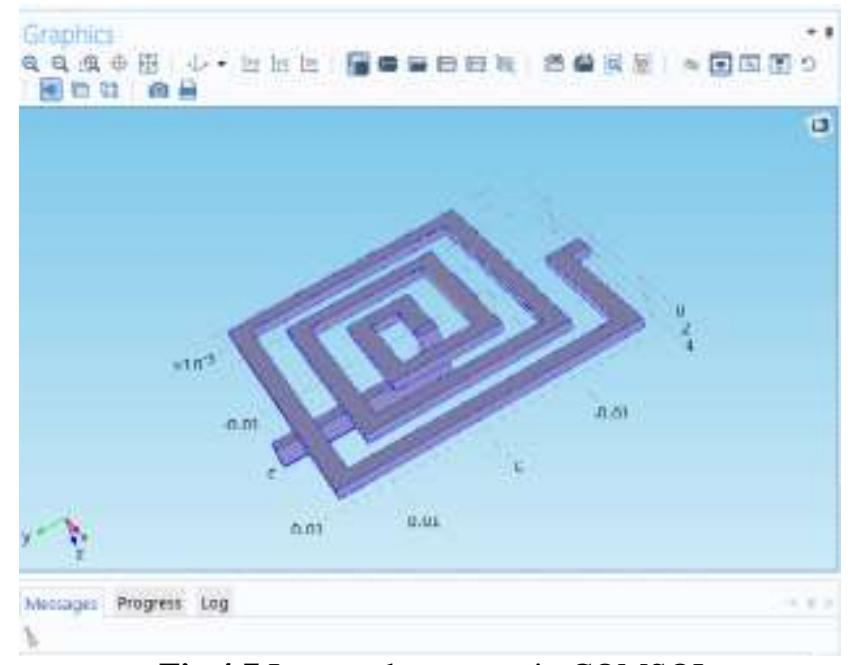

Fig 4.7 Imported structure in COMSOL

The self-inductance of the inductor is given as

$$
L=\frac{2 * \mathrm{Wm}}{\mathrm{I} * \mathrm{I}}
$$

Where'Wm' is the magnetic energy and ' $\mathrm{I}$ ' is the current. The model uses the Terminal boundary condition, whichsets the current to $1 \mathrm{~A}$ and automatically computes theselfinductance. The self-inductance $\mathrm{L}$ becomes available as the $\mathrm{L}_{11}$ component of the inductance matrix. [6]

\section{SIMULATION RESULTS \& ANALYSIS}

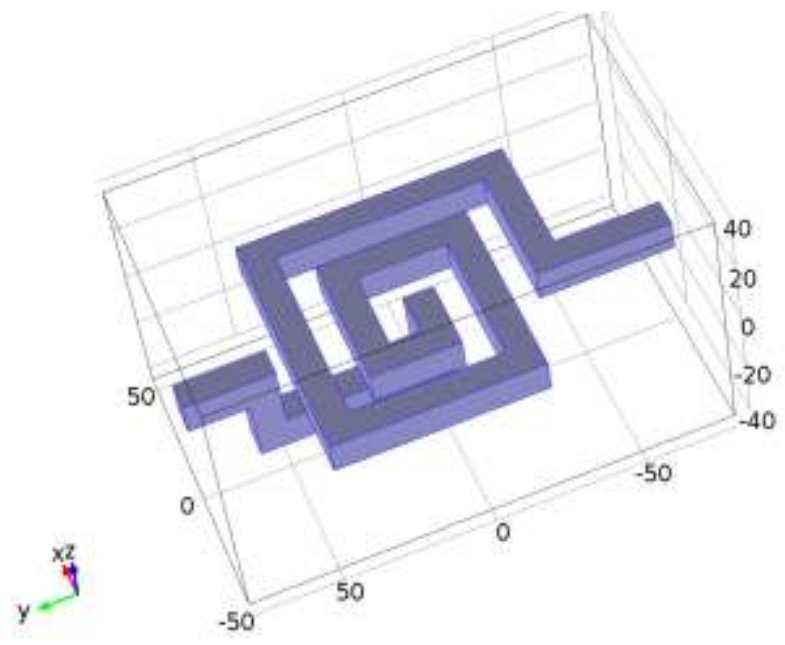

Fig 5.1Geometry of the inductor

The figure 5.1 shows the geometry of the inductor which was imported.

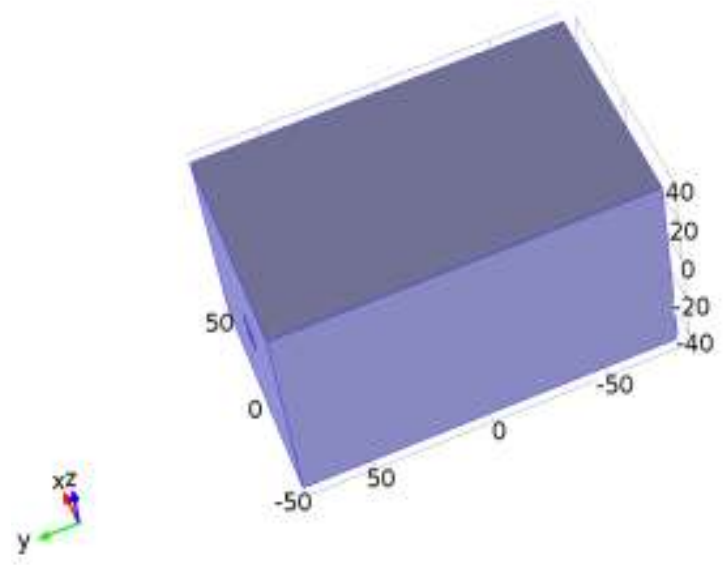

Fig 5.2 Geometry of the surrounding boundary

The figure 5.2 shows the geometry of the surrounding layer of air. The edge of the inductor can be seen in the middle.

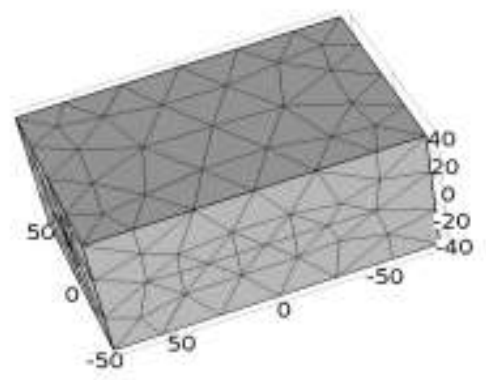

Fig 5.3 Meshing of the entire structure 
Figure 5.3 shows the meshed structure of the model which is developed after the simulation has been completed. The meshing used here is physics controlled mesh with coarse element size.

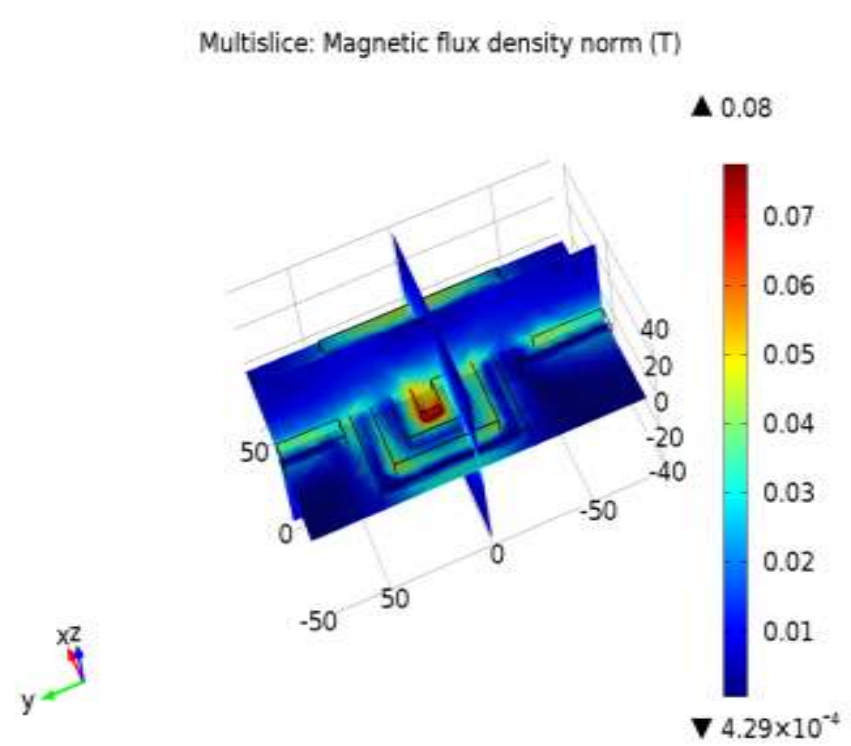

Fig 5.4Magnetic flux density around the device

Figure 5.4 shows the normalized flux density in Tesla which is developed around the entire structure. The flux density is the highest in the middle of the inductor.

Surface: Electric potential ( $V$ )

mline: Magnetic flux density Streamline Color: Magnetic flux density no

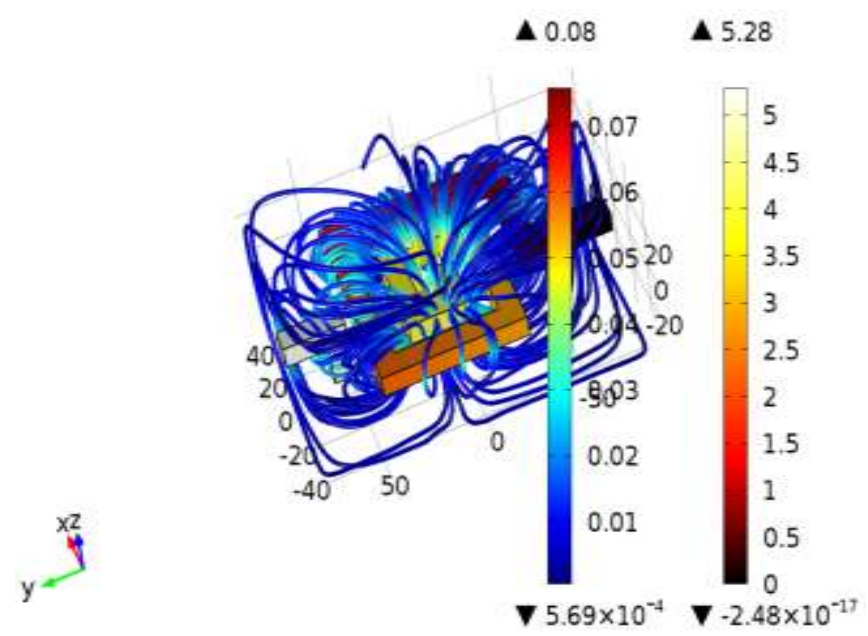

Fig 5.5Surface: Electric potential (V) Streamline: Magnetic flux lines around the device

Figure 5.5 shows the shows the electric potential in the inductor and the magnetic flux lines. The color of the flow lines represents the magnitude of the magnetic flux. As expected this flux is largest in the middle of the inductor.

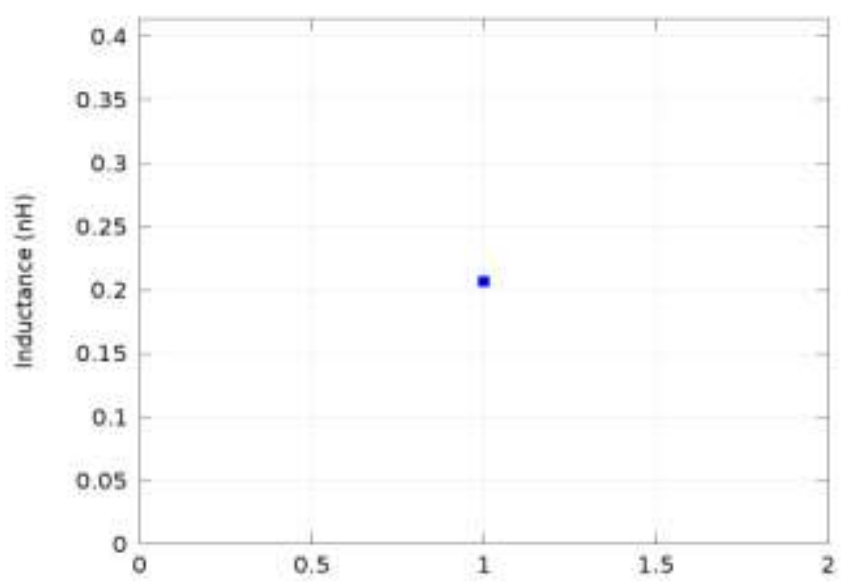

Fig 5.6Inductance value in $n H \&$ frequency

Figure 5.6 shows the 1-D curve between the inductance value and the frequency. The inductance value calculated for the structure is $0.0206 \mathrm{nH}$.

Table -1: Comparative Result from other works

\begin{tabular}{|l|l|l|l|l|}
\hline Work & $\begin{array}{l}\text { Type of } \\
\text { Result }\end{array}$ & $\begin{array}{l}\text { Freq } \\
(\mathrm{GHz})\end{array}$ & $\begin{array}{l}\mathrm{L} \\
(\mathrm{nH})\end{array}$ & Size $\left(\mu \mathrm{m}^{2}\right)$ \\
\hline$[3]$ nitride & simulation & 1 & 2.4 & $315 * 315$ \\
\hline$[3]$ oxide & simulation & 1 & 2.6 & $315 * 315$ \\
\hline$[4]$ & measurement & 11 & 1.4 & $216 * 228$ \\
\hline$[5]$ & simulation & 2.5 & 6 & $265 * 265$ \\
\hline This work & simulation & 1 & 0.20 & $100 * 160$ \\
\hline
\end{tabular}

\section{CONCLUSION}

The MEMS devices are continuously undergoing the change. With respect to recent technology and process, it can be said that to catch up with the current trend where the device size is now shrinking to nanometers, and to catch up with the current time to market ratio, rigorous testing is required. This testing is required within minimum possible time. Hence, co-simulation is demand of time and that will reduce the dependency of on one platform for solving complex structures.

Hence, we proposed a methodology to design by using SOLIDWORKS and COMSOL Multiphysics. We have designed MEMS spiral inductor using this method which has the self inductance value as $0.20 \mathrm{nH}$ and the area. The dimension of the inductor is $100 * 160 \mu \mathrm{m}^{2}$ which occupies less area than the other works.

\section{FUTURE PLAN}

This methodology of co-simulation can be extended for the designing of the MEMS devices having complex design. By this process of co-simulation, it is possible to use the effective designing techniques present with the SOLIDWORKS and effective simulating techniques present in COMSOL Multiphysics.

In future the electronic Interface with the proposed inductorused in various bio MEMS applications such as blood pressure monitoring etc. can be implemented [8-9]. 


\section{REFERENCES}

[1]. Guan Hue "RF spiral planer inductor designs preliminaryResult" Faculty of Electrical Engineering, university technology Malaysia.

[2]. J.N. Burghartz, D.C. Edelstein, M. Soyuer, H.A. Ainspan and K.A.Jenkins, "RF circuit design aspects of spiral inductors on silicon," IEEE J.Solid-State Circuits, vol. 33, no. 12, pp. 2028-2034, Dec. 1998.

[3]. Lee, J., S. Park, H. C. Kim, and K. Chun, "Substrates and dimension dependence of MEMSinductors," IOP Science Journal of Micromechanics and Microengineering, Vol. 19, 085014, 2009.

[4]. Dai, C.L., J.Y. Hong andM.C. Liu, "High Q-factor CMOS-MEMS inductor," Symposium on Design, Test, Integration and Packaging of MEMS/MOEMS, 138-141, Apr. 2008.

[5]. Gil, J. and H. Shin, "A simple wide-band on-chip inductor model for silicon-based RF ICs," IEEE Transactions on Microwave Theory Techniques, Vol. 51, No. 9, 2023-2028, Sep. 2003.

[6]. https://www.comsol.co.in

[7]. Sia, C. B., W. M. Lim, B. H. Ong, A. F. Tong, and K. S. Yeo, Modeling and layout optimizationtechniques for silicon-based symmetrical spiral inductors," Progress In Electromagnetics Research, Vol. 143, 1-18, 2013.

[8]. Peng Cong, Wen H. Ko, Darrin J. Young "IntegratedElectronic System Design for an Implantable WirelessBatteryless Blood Pressure Sensing Microsystem"IEEE Communications Magazine pp. 98-104, April2010.

[9]. Darrin J. Young "Interface Electronics for MEMSBasedWireless Sensing Applications" InternationalSymposium on VLSI Design Automation and Test(VLSI-DAT), pp. 130 - 133,Hsin Chu, 26-29 April2010.

\section{BIOGRAPHIES}

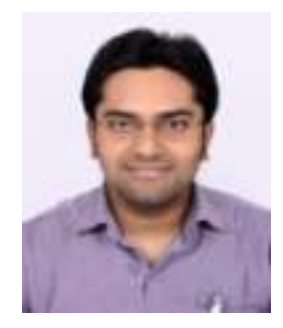

Deepak Kumar Sinha is currently pursuing M.E in Electronics \& Telecommunication Engineering degree with Thakur College of Engineering \& Technology, Mumbai University, India. His current research interests include the MEMS, VLSI Design and Nanotechnology.

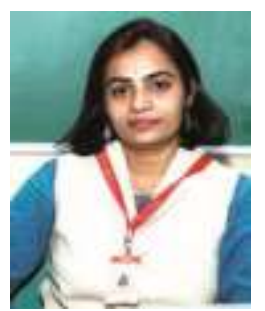

Dr. Sandhya Save received the Ph.D. degreefrom SNDT University, Mumbai, India in 2015.Her research interests include VLSI Design, Analog and mixed signal system and Electronic Devices. She is currently HOD and Professor in Electronics Engineering Department at Thakur College of Engineering \& Technology, Mumbai, India. 